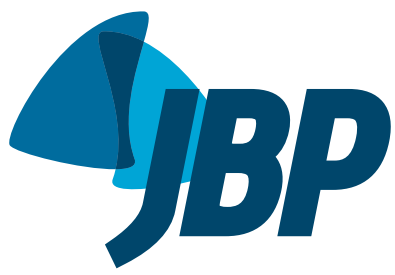

\title{
BCGitis: A rare complication after intravesical BCG therapy
}

\author{
Maria João Oliveira', Daniel Vaz¹, Aurora Carvalho ${ }^{1,2}$, Rosário Braga ${ }^{3}$, \\ Raquel Duarte ${ }^{1,2,4,5}$
}

\section{DEAR EDITOR:}

In most cases, bladder carcinoma is located superficially, and the therapeutic approach is usually transurethral resection followed by intravesical therapy (chemotherapy or immunotherapy). ${ }^{(1,2)}$ Initially produced as a vaccine against tuberculosis, BCG-an attenuated strain of Mycobacterium bovis-has been widely used in immunotherapy over the last decades. ${ }^{(1,3)}$ Although BCG, used as immunotherapy, has not produced the best results in many cancers, it has been clinically successful in the intravesical treatment of superficial bladder carcinomas. ${ }^{(4)}$ Immunotherapy with intravesical BCG eradicates the residual tumor, slows the progression of the disease, reduces the need for cystectomy, and prolongs survival. ${ }^{(4)}$ Treatment with BCG is well tolerated by over $95 \%$ of patients. The most common side effects are local (inflammation, fever, and pelvic adenopathy). However, although rare, systemic complications have been described. ${ }^{(1,3-6)}$ Systemic dissemination of the attenuated $M$. bovis bacillus is known as BCGitis. It is more common in individuals with underlying primary or secondary immunodeficiency but can occur in immunocompetent patients. ${ }^{(1,6)}$ There have been only a few reports of cases of respiratory BCGitis. ${ }^{(1,2,6)}$ Here, we report the case of a patient who was treated with local BCG immunotherapy for urothelial bladder carcinoma and developed a severe $M$. bovis respiratory infection.

In March of 2013, a 72-year-old male presented with hematuria. The patient also had hypertension, dyslipidemia, cerebrovascular disease (previous stroke), chronic renal failure, and depression. He was being treated with perindopril, indapamide, atorvastatin, acetylsalicylic acid, furosemide, and escitalopram. He had no known drug allergies. Ultrasound revealed a $2.5-\mathrm{cm}$ polyp in the bladder. The patient underwent transurethral resection of the bladder tumor. Histological examination revealed urothelial cell carcinoma (grade 2), without vascular or muscle invasion. Chemotherapy and immunotherapy with intravesical instillation of BCG were started and continued until December of 2013. In February of 2014, the patient presented to the emergency department with a one-week history of dyspnea and productive cough (mucopurulent sputum). He reported no fever, chest pain, hemoptysis, sweating, or other symptoms. On physical examination, he was afebrile, with an increased respiratory rate, hemodynamic stability, and an $\mathrm{SpO}_{2}$ of $89 \%$ on room air. Auscultation revealed bilateral breath sounds, with crackles in both lung bases. There were no other significant changes. Ancillary tests showed a C-reactive protein level of $10 \mathrm{mg} / \mathrm{dL}$, without leukocytosis, together with normal liver and kidney function, overlapping with previous studies of the patient. Blood gas analysis (at a $\mathrm{FiO}_{2}$ of 24\%) showed a pH of 7.43, a $\mathrm{PaO}_{2}$ of $66 \mathrm{mmHg}$, a $\mathrm{PaCO}_{2}$ of $45 \mathrm{mmHg}$, and an $\mathrm{HCO}_{3}$ of $29.9 \mathrm{mEq} / \mathrm{L}$. In addition, a chest X-ray showed bilateral diffuse reticulonodular infiltrates. A CT scan of the chest revealed randomly distributed micronodules in the lungs, together with enlargement of the hilar and mediastinal lymph nodes (Figure 1). After having collected sputum for analysis, we started empirical antibiotic treatment with amoxicillin and clavulanic acid. Due to respiratory failure and suspected miliary tuberculosis or diffuse pulmonary metastasis, the patient was admitted to the respiratory ward of the hospital.

During hospitalization, microbiological and mycobacteriological cultures of sputum samples were negative. Bronchoscopy revealed bilateral mucopurulent secretions, edema, and diffuse mucosal congestion. Microbiological cultures of bronchial lavage and BAL fluid (BALF) samples were negative. Although the BALF was negative for $M$. tuberculosis on smear microscopy, it tested positive for M. tuberculosis complex DNA and for M. bovis in cultures. Cytology of the BALF was negative for malignant cells; the patient also tested negative for the viral markers

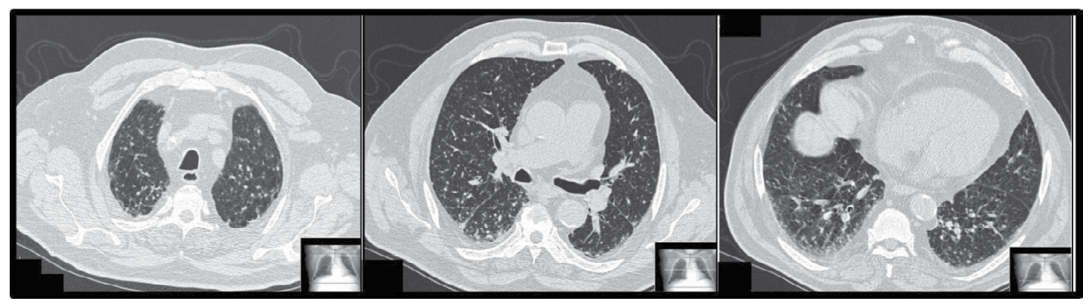

Figure 1. Chest CT scan showing randomly distributed micronodules in the lungs, together with enlargement of the hilar and mediastinal lymph nodes.

1. Serviço de Pneumologia, Centro Hospitalar de Vila Nova de Gaia/Espinho, Vila Nova de Gaia, Portugal.

2. Centro de Diagnóstico Pneumológico, Vila Nova de Gaia, Portugal.

3. Serviço de Patologia Clínica, Centro Hospitalar de Vila Nova de Gaia/Espinho, Vila Nova de Gaia, Portugal.

4. Unidade de Investigação em Epidemiologia - EPIUnit - Instituto de Saúde Pública, Universidade do Porto, Porto, Portugal.

5. Departamento de Epidemiologia Clínica, Medicina Preditiva e Saúde Pública, Faculdade de Medicina, Universidade do Porto, Porto, Portugal. 
of infection with HIV, HCV, and HBV. Because of the positive result on the nucleic acid amplification test of the BALF, we started tuberculosis treatment with isoniazid, rifampin, pyrazinamide, and ethambutol. Despite improvements in his test results and clinical resolution of the respiratory failure, the patient showed a slight increase in liver enzymes (to less than double the normal values). He underwent upper abdominal ultrasound, which revealed hepatic steatosis with no other abnormalities. In view of the BALF culture positivity for M. bovis (which is naturally pyrazinamide-resistant), we suspended the pyrazinamide, and the liver enzyme values normalized thereafter.

The patient was discharged and continued treatment on an outpatient basis. Future scheduled BCG instillations were suspended. In the initial phase of treatment, the regimen was the isoniazid-rifampin-ethambutol combination, whereas the isoniazid-rifampin combination was used in the maintenance phase. After a total of 6 months of treatment, the patient showed a good response. At this writing, the patient remains under follow-up monitoring of his bladder, without signs of recurrence. The patient gave written informed consent for the reporting of his case.
Various recent studies have analyzed the outcomes of patients with bladder carcinoma who underwent therapeutic intravesical instillation of BCG. The study conducted by Lamm et al. showed that disseminated BCGitis was extremely rare. (2) Some studies have reported that, among patients with bladder cancer, there is a relationship between the complications of BCG instillation and a prior diagnosis of tuberculosis, such complications not occurring in patients without a history of tuberculosis and with no evidence of sequelae on chest X-rays. ${ }^{(1)}$

In this report, we have presented the case of a male patient without a history of tuberculosis who developed a respiratory infection soon after treatment with intravesical instillation of BCG. The initial diagnostic hypotheses included malignancy. However, after careful investigation, the patient was diagnosed with BCGitis, with no remaining doubt that the lung disease was due to the spread of the BCG used in the treatment of his bladder carcinoma. Despite the rarity of this complication, our case report underscores the need for vigilance and awareness of the possibility of BCG dissemination, given the widespread use of BCG in patients with bladder carcinoma.

\section{REFERENCES}

1. Manfredi R, Dentale N, Piergentili B, Pultrone C, Brunocilla E. Tubercular disease caused by bacillus of Calmette-Guerin administered as a local adjuvant treatment of relapsing bladder carcinoma. Pathogenetic, diagnostic and therapeutic issues, and literature review. AVFT [serial on the Internet]. 2009 Jul [cited 2015 Jun 15];28(2):54-60. Available from: http://www.revistaavft.com/ avft\%202\%202009/hoja4.html

2. Lamm DL, van der Meijden PM, Morales A, Brosman SA, Catalona WJ, Herr HW, et al. Incidence and treatment of complications of bacillus Calmette-Guerin intravesical therapy in superficial bladder cancer. J Urol. 1992;147(3):596-600.

3. Harbjerg JL, Bierre CC, Lillebæk T, Weinreich UM, Pulmonal bacillus Calmette-Guérin infection two years after intravesical bacillus
Calmette-Guérin installation [Article in Danish]. Ugeskr Laeger 2014;176(25A) pii: V07120381.

4. de Saint Martin L, Boiron C, Poveda JD, Herreman G. Generalized BCG infection after intravesical instillations of Calmette-Guérin bacillus [Article in French]. Presse Med. 1993;22(29):1352-6.

5. Sicard D, Steg A, Leleu C, Boccaccio F, Abadia R, Tulliez M, et al "BCGitis", a systemic complication of intravesical BCG therapy of bladder tumor [Article in French]. Ann Med Interne (Paris). 1987;138(7):555-6.

6. Deeks SL, Clark M, Scheifele DW, Law BJ, Dawar M, Ahmandipour $N$, et al. Serious adverse events associated with bacille CalmetteGuérin vaccine in Canada. Pediatr Infect Dis J. 2005;24(6):538-41. http://dx.doi.org/10.1097/01.inf.0000164769.22033.2c 